\title{
Grating-coupled surface plasmons at microwave frequencies
}

\author{
A. P. Hibbins ${ }^{\text {a) }}$ and J. R. Sambles \\ Thin Film Photonics Group, School of Physics, University of Exeter, Exeter, EX4 4QL, England \\ C. R. Lawrence \\ Defence Evaluation and Research Agency, Farnborough GU14 OLX, England
}

(Received 17 November 1998; accepted for publication 26 April 1999)

\begin{abstract}
This work presents a detailed investigation of electromagnetic coupling to the surface plasmon-polariton (SPP) at microwave frequencies. We have recorded the wavelength-dependent reflectivity from a metallic sinusoidal diffraction grating of pitch $15 \mathrm{~mm}$. In order to minimize the problems associated with nonplanar incident wavefronts, we have developed an apparatus that collimates the incident beam. We illustrate resonant coupling to the SPP at wavelengths of the order of $10 \mathrm{~mm}$. The wavelength-dependent reflectivities recorded have been successfully fitted using a differential formalism of conical diffraction with a single set of grating parameters describing the grating profile and metal permittivity. (C) 1999 American Institute of Physics.
\end{abstract}

[S0021-8979(99)08215-8]

\section{INTRODUCTION}

The electromagnetic surface waves discussed in this work propagate along an interface between two dissimilar media without radiation losses. Surface waves ${ }^{1,2}$ have attracted much scientific attention over the last one hundred years. For example, Zenneck ${ }^{3}$ in 1907 realized the possibility of propagation of radio waves around the Earth when one considers one half-space as a pure dielectric and the other as a conductor. A similar wave of radiation coupled to oscillating charge density may propagate along a metal-dielectric interface. We refer to this mode as a surface plasmonpolariton (SPP) and its influence on the reflectivity from metallic gratings may be realized in the visible part of the spectrum as a Wood's anomaly. ${ }^{4-7}$

Because the real parts of the dielectric functions of the media either side of the interface are of opposite signs, the normal components of the electric field at the metaldielectric interface oppose each other. Hence the surface mode is strongly localized at the surface with exponentially decaying fields into the adjacent media. The propagation of the SPP along the interface is then extremely sensitive to the dielectric permittivities of the media surrounding it. ${ }^{6}$ However, the dispersion relation of a SPP on a planar surface ${ }^{5}$ (Fig. 1) is such that the wave vector of the mode is always greater than the maximum photon wave vector available in the adjacent dielectric. Hence, on a flat surface of infinite extent, the SPP is unable to radiate or be directly coupled to by incident planar waves. Resonant interaction of electromagnetic radiation with the surface may only be achieved by increasing the wave vector of the incident radiation, possibly by using a high-index prism ${ }^{8,9}$ or through surface roughness. The latter is most commonly found in the form of a diffraction grating, where the periodic corrugation provides the necessary in-plane wave-vector enhancement in multiples of the grating wave-vector $k_{g}=2 \pi / \lambda_{g}$. This gives rise to diffracted

\footnotetext{
${ }^{a)}$ Electronic mail: a.p.hibbins@exeter.ac.uk
}

orders which no longer propagate in the dielectric when their in-plane wave vector is increased such that it becomes greater than that of the incident radiation. It is the enhanced wave vector of these evanescent fields that allows incident radiation to couple to the SPP according to the condition

$$
\mathbf{k}_{\mathrm{SPP}}=\left(n_{d} k_{0} \sin \theta\right) \hat{\mathbf{x}} \pm N \mathbf{k}_{g} .
$$

Here, $N$ is an integer and $n_{d} k_{0} \sin \theta$ is the projection of the wave vector of the incident radiation along the $x$ axis (Fig. 2 ). If the angle between the plane of incidence and the grating wave vector (called the azimuthal angle, $\varphi$ ) is equal to zero, then all the diffracted beams lie in the plane of incidence and Eq. (1) reduces to a scalar equation.

Variation of the magnitude of the incident wave vector in the plane of the grating may be achieved by scanning either the wavelength $\left(\lambda_{0}\right)$, or angle of incidence $(\theta$ or $\varphi)$. Hence, provided that there is a component of the electric field normal to the interface in order to create the necessary surface charge, and the grating is of suitable pitch, wave vector values will exist at which radiation may couple resonantly to the SPP mode. This SPP may then reradiate into any propagating order or it may be absorbed. The subsequent loss of intensity from the specular reflectivity is recorded as a function of wavelength and then compared with that predicted by a rigorous grating theory model, which uses a conical version ${ }^{10}$ of the differential formalism of Chandezon et al. ${ }^{11}$ This allows one to characterize the surface profile of the grating ${ }^{12}$ and the dielectric properties of the media either side of the interface. ${ }^{13}$

Many previous workers have studied extensively the grating-coupled SPP mode at visible ${ }^{5,12,13}$ and infrared ${ }^{14}$ wavelengths on corrugated surfaces produced by standard interferographic techniques. ${ }^{15}$ Before this method of grating manufacture was established, gratings had to be ruled by hand, ${ }^{4}$ hence experimentalists often made use of the larger scale of systems available in the microwave regime. For example, in 1957, Meecham and Peters ${ }^{16}$ studied the reflection of radiation of wavelength $\lambda_{0}=32 \mathrm{~mm}$ from an echelette 


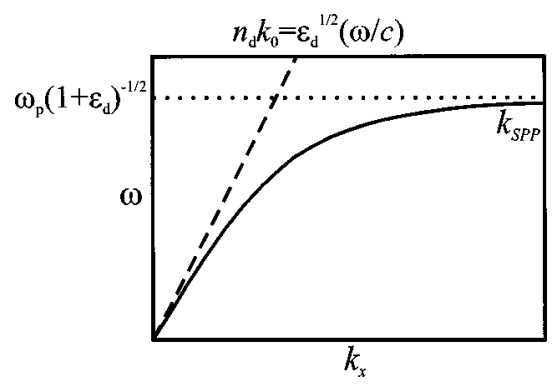

FIG. 1. Schematic dispersion curve for a SPP on a planar metal-dielectric interface, $k_{\mathrm{SPP}}=k_{0} \sqrt{\left(\epsilon_{m} \epsilon_{d} / \epsilon_{m}+\epsilon_{d}\right)}$, where $\epsilon_{d}=n_{d}^{2}$ and $\epsilon_{m}$ are the permittivities of the dielectric and metallic media, respectively, $k_{\mathrm{SPP}}$ is the wave vector of the surface mode, $k_{0}=\omega / c$ is the wave vector of the incident radiation and $\omega_{p}$ is the plasma frequency of the metal. The light line for a grazing photon is also shown $\left(\omega=n_{d} k_{0}\right)$. Since these grazing photons have the maximum possible value of $k_{x}$ (along the interface), it is not possible to couple directly to the surface mode. However, note that in the low frequency (microwave) limit, $k_{\mathrm{SPP}} \rightarrow n_{d} k_{0}$.

grating with the electric field of the radiation parallel to the grooves. As they scanned the angle of incidence, they observed a redistribution of energy among the diffracted beams at the point at which an order begins, or ceases to propagatethe pseudocritical edge or Rayleigh anomaly. ${ }^{17}$ They compared their experimental reflectivities from a finite grating (15 elements) to a theory based on a variational technique ${ }^{18}$ which, like many other diffraction theories developed around this time, assumed the surface to be of infinite extent and perfectly conducting $\left(\epsilon_{r}=-\infty\right)$. This is a valid assumption to make since the frequency of the impinging microwave radiation is many orders of magnitude less than the plasma frequency, and the effect of any absorption in the metal will be insignificant due to the negligible skin depth. Hence at these wavelengths, all metals may be treated as nearperfectly conducting.

In 1965, Harvey Palmer et al. ${ }^{19}$ reported measurements for the spectral distribution of radiation of wavelength $4 \mathrm{~mm}$ from metal gratings of rectangular profile. When the wave propagation vector was lying in a plane normal to the grating grooves, with the electric field vector perpendicular to the grooves, dark anomalies were observed that became wider as the groove depth was increased. An attempt was made to

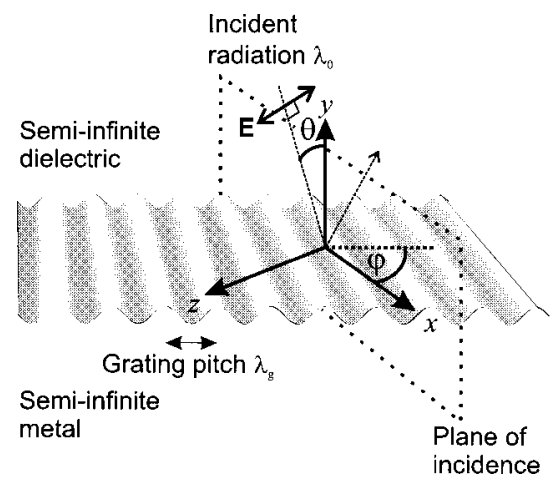

FIG. 2. The coordinate system describing the experimental geometry and plane of incidence with respect to the direction of the grating grooves. The direction of the electric field vector $\mathbf{E}$ is illustrated for the situation when $p$-polarized [transverse magnetic (TM)] radiation is incident. explain this phenomenon using a theory based on "surface wave modes which are supported by the rectangular periodic structure of the grating", however no illustration of these results is presented.

It is well known that the propagation length of the SPP mode along the interface, and hence the width of the SPP resonance, is governed by two damping processes: ${ }^{5,20}$ the absorption of energy in the metal substrate (which is proportional to the imaginary part of the dielectric function of the metal, $\left.\epsilon_{i}\right)$, and scattered radiative losses into the dielectric media (which is proportional to $a^{2}$ to first order, where $a$ is amplitude of the grating ${ }^{21}$ ). Note that the existence of this radiation loss does not invalidate the previous definition of the surface wave since it is dependent only on the surface profile and not the properties of the adjacent media. Hence, for shallow gratings with almost no scattering of radiation into diffracted orders, experimental observation of the SPP resonance is only possible if the metallic medium is lossy (e.g., a corrugated gold surface, ${ }^{12} a / \lambda_{g} \approx 0.05, \epsilon_{\lambda_{0}}=632.8 \mathrm{~nm}$ $=-10.8+1.3 i)$. As previously discussed, at microwave frequencies, all metals behave as near-perfect conductors and hence the direct Joule-heating loss is insignificant. However, as the amplitude of the corrugation is increased, the probability of the surface wave being scattered into a diffracted order also grows. In other words, the propagation length of the SPP on a highly corrugated surface will be less than that on a shallow grating. Hence, a propagating surface wave on a highly perturbed surface will not sample an infinite number of grooves, instead it will be scattered by a range of grating wave vectors determined by the Fourier transform of such a finite set of corrugations. This will introduce a range of angles over which the SPP may reradiate into the specular or one of the diffracted orders, and hence an angular width will be associated with the resonance as measured by the loss of reflectivity in the zero order beam. In addition to the widening of the SPP resonance, we also expect the momentum of the resonance to increase due to perturbation of the surface away from the planar case., ${ }^{5,22-24}$ The work of Palmer et al. ${ }^{19}$ shows such a change in the shape of a resonance, and we believe that work provided the first experimental evidence of the propagation of an electromagnetic surface mode, the SPP, on a near-perfectly conducting surface. It is important to note, however, that the resonance of the mode may only be observed when there is another channel into which energy may be transferred. There are up to three ways in which this may occur. Firstly, Joule heating of any absorbing media in the system will take place. Second, energy from the mode may be reradiated into any propagating diffracted orders in the system. This is a similar effect to that modeled by Kitson et $\mathrm{ll}^{25}$ who observed an increase in the width of the resonance when the SPP mode is able to couple to a propagating diffracted order. The diffracted order provides a strong radiative decay channel for the mode which contributes to its damping, and hence width. Finally, reradiation of energy from the mode back into the specular beam may occur. If the grating grooves are neither parallel nor perpendicular to the plane of incidence $\left(\varphi \neq 0,90^{\circ}\right)$, the phase of this radiation will be rotated with respect to the incident beam and a polarization conversion signal may be detected. 


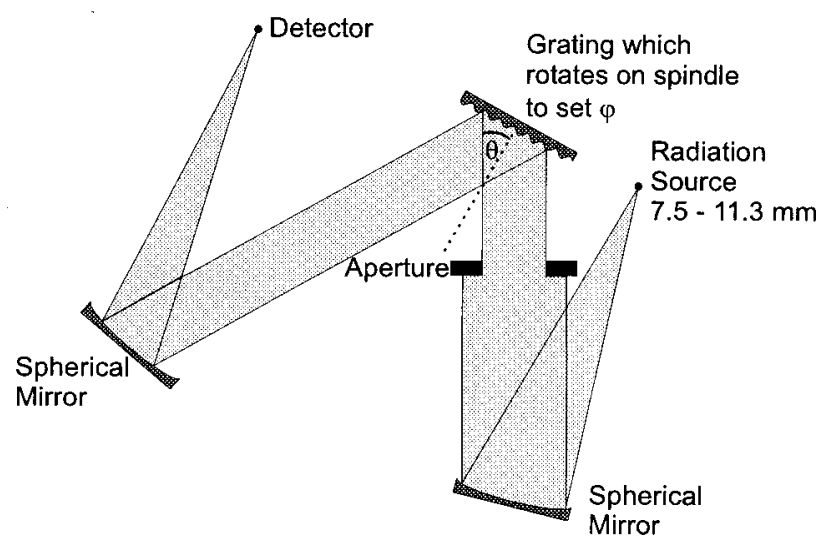

FIG. 3. Schematic diagram illustrating the apparatus used to measure the wavelength-dependent response from corrugated test samples.

This article presents a detailed experimental study of the grating-coupled SPP excited by electromagnetic radiation in the microwave regime. As mentioned previously, at these wavelengths, all metals behave as near perfect conductors and the dispersion of the SPP is dependent only on the interface profile of the sample.

\section{EXPERIMENT}

Figure 3 illustrates the experimental arrangement used to record the reflectivity from the sample as a function of wavelength, and Fig. 4 shows a schematic representation of the interconnecting components of the system.

The arrangement shown in Fig. 3 is similar to that of the Czerny-Turner spectrometer ${ }^{26}$ and has been developed in order to reduce the undesirable effects of spherical wavefronts which will provide excitation of the SPP even without the grating. The problem of near-spherical wavefronts arises from the standard gain horn geometry and the large radiation wavelength. Typically the beam divergence is some $20^{\circ}$. By placing the transmitting horn at the focus of a $2 \mathrm{~m}$ focal length mirror, the incident beam is well collimated. A circular, metallic aperture is also used to reduce the width to give a well-collimated beam. A second mirror is positioned to collect the specularly reflected beam from the grating and focus it into the detector. The aluminum-alloy mirrors have been milled with a diameter of $450 \mathrm{~mm}$ and have a radius of curvature, $R=4 \mathrm{~m}$.

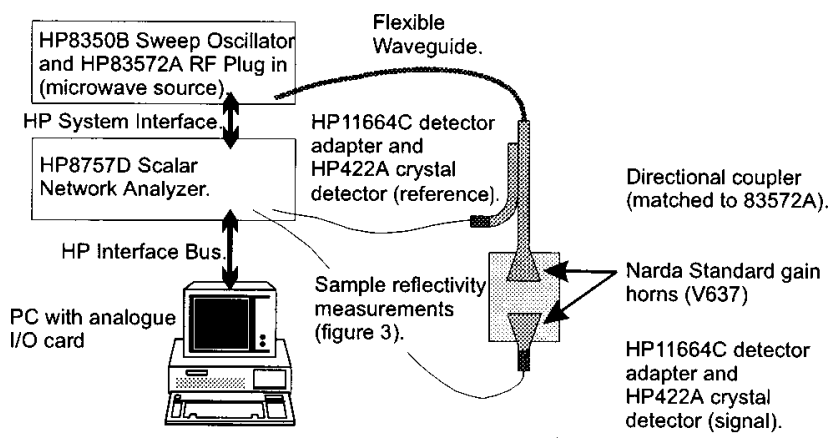

FIG. 4. Schematic representation of the interconnecting components used to record reflectivities in the $7.5-11.3 \mathrm{~mm}$ wavelength range.

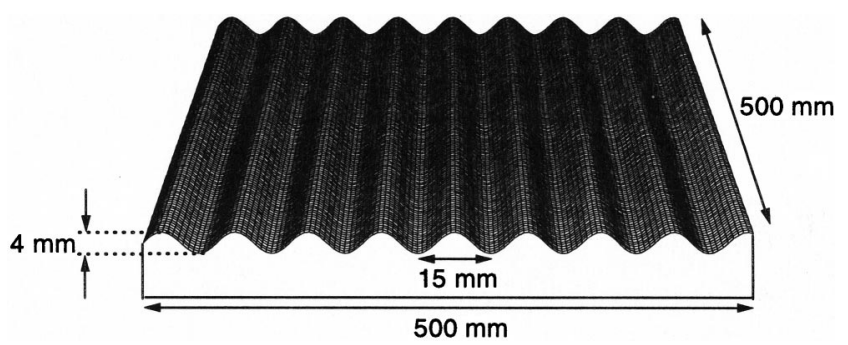

FIG. 5. Schematic diagram of the aluminum-alloy grating used in this study.

Data are recorded as a function of wavelength between 7.5 and $11.3 \mathrm{~mm}$ at a polar angle of incidence of approximately $\theta=31^{\circ}$, and a series of azimuthal angles $(\varphi)$ every $15^{\circ}$ between $0^{\circ}$ and $90^{\circ}$. (The azimuthal angle $\varphi$ is defined as the angle between the plane of incidence and the grating vector, $k_{g}=2 \pi / \lambda_{g}$.) These angles are set to a precision of $\pm 1^{\circ}$. The transmitting and receiving horn antenna are set to pass either $p$ [transverse magnetic (TM)], or $s$ [transverse electric (TE)] polarizations, defined with respect to the plane of incidence. This enables the measurement of $R_{p p}$, and $R_{s s}$, and polarization conversion ${ }^{12,27} R_{s p}$, and $R_{p s}$ reflectivities where the subscripts refer to the incident and detected polarizations in that order. To account for any fluctuations in the power of the source, the output from the signal detector is divided by that of the reference, and the resulting wavelength-dependent data were normalized by comparison with the reflected intensity from a flat metal plate.

For each angle of incidence, the reflectivity data were fitted to the grating modeling theory. The theory uses a scattering matrix technique ${ }^{28}$ based on a conical version ${ }^{10}$ of the differential formalism of Chandezon et al. ${ }^{11}$ It involves the transformation of the mathematics into a new, nonrectilinear coordinate system in which the boundary conditions at the corrugated interface are more readily solved, effectively flattening the surface. This requires that the field amplitudes are represented as a Fourier expansion. The surface itself is also described by a Fourier series:

$A(x)=a_{1} \cos \left(k_{g} x\right)+a_{2} \cos \left(2 k_{g} x\right)+\ldots+a_{N} \cos \left(N k_{g} x\right)+\ldots$,

where $a_{N}$ provides the first-order scattering mechanism to the $\pm N$ SPP. By fitting the reflectivity data to the modeling theory, assuming that the metal substrate is perfectly conducting, it is possible to accurately parametrize the grating profile.

The grating studied in this work has been manufactured from the same alloy as the mirrors, using a computer-aided design and manufacture technique with a $6 \mathrm{~mm}$ diameter ball-ended slot drill. The mill was programmed to produce a purely sinusoidal profile, with a pitch of $\lambda_{g}=15 \mathrm{~mm}$ and amplitude of $a_{1}=2 \mathrm{~mm}$ (Fig. 5).

\section{RESULTS}

Figure 6 shows four typical sets of experimental data $(+)$ at azimuthal angles of $\varphi=30^{\circ}, 45^{\circ}, 60^{\circ}$, and $75^{\circ}$ showing the selected reflectivities $R_{p p}, R_{s p}, R_{p s}$, and $R_{s s}$, re- 

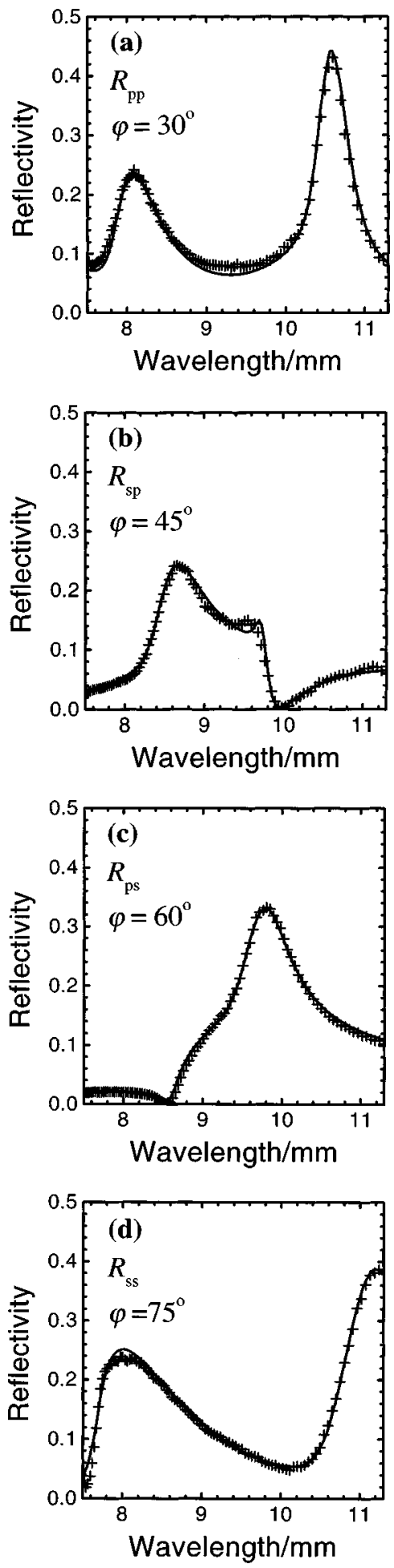

FIG. 6. The normalized (a) $R_{\mathrm{pp}}$, (b) $R_{\mathrm{sp}}$, (c) $R_{\mathrm{ps}}$, and (d) $R_{\mathrm{ss}}$ experimental wavelength-dependent signals $(+)$ compared with the theoretically modelled results (-) created from a single set of profile and metal-permittivity parameters. Radiation is incident on the test sample at polar angle $\theta=31^{\circ}$ and at azimuth angles of (a) $\varphi=30^{\circ}$, (b) $\varphi=45^{\circ}$, (c) $\varphi=60^{\circ}$, and (d) $\varphi=75^{\circ}$. The angles quoted are approximate $\left( \pm 1^{\circ}\right)$, and only one point in four is shown for clarity.

spectively. The solid curves are the theoretical fits, which are in excellent agreement with the experimental data.

In an ideal system the wavefronts incident on the sample would be completely planar. However, the radiation source is finite and the source antenna and aperture diffract the beam. Hence, in order to achieve this quality of fits, a degree of Gaussian beam spread had to be introduced into the theoretical modeling. We have assumed a simple linear relationship between wavelength and incident-angle spread since the diffraction effects will clearly increase with wavelength. We also find that the angle spread is dependent on the orientation (i.e., polarization) of the rectangular transmitting and receiving antennas. Typically, at the center of the wavelength range, the standard deviation of Gaussian angle spread is $\sigma(\theta) \approx 0.9^{\circ}$. This represents a dramatic improvement over the uncollimated system $\Delta \theta \sim 20^{\circ}$, but it is still large enough to significantly affect the measured reflectivities.

The theoretically modeled data were fitted to the experimental data using the angles of incidence $(\theta$ and $\varphi)$, the above linear variation of beam spread $[\sigma(\theta)]$, and the grating profile $\left(a_{1}, a_{2}, a_{3}\right.$, and $\left.a_{4}\right)$ as fitting parameters. The profile of the grating is initially assumed to be purely sinusoidal, and the angles of incidence are allowed to vary in each scan by up to $1^{\circ}$ from their measured values. We then introduce a small amount of distortion to the profile to further improve the fits, finally adjusting the degree of beam spread to account for the finite source size and diffraction in the system. The grating profiles determined for each wavelength scan are averaged and the error about the mean calculated. The final fits shown are then all generated from this averaged set of profile parameters: $a_{1}=2.01 \pm 0.01 \mathrm{~mm}, \quad a_{2}=-0.02$ $\pm 0.02 \mathrm{~mm}, a_{3}=0.02 \pm 0.01 \mathrm{~mm}$, and $a_{4}=-0.01 \pm 0.01 \mathrm{~mm}$ with a grating pitch of $\lambda_{g}=14.95 \mathrm{~mm}$. The permittivities were taken to be $\epsilon_{d}=n_{d}^{2}=1.0$ and $\epsilon_{m}=\epsilon_{r}+i \epsilon_{i}=-10^{6}$ $+10^{6} i$ for the dielectric (air) and metal, respectively.

\section{DISCUSSION}

This work has demonstrated that it is possible to couple microwave radiation to a SPP that propagates on a nearperfectly conducting metal-dielectric interface. Figure 6 illustrates that by using the rigorous diffraction modeling theory we are able to produce high-quality fits to the experimental data allowing the grating profile to be characterized. The grating profile has also been determined by scanning a dial gauge, sensitive to $0.025 \mathrm{~mm}$, across the metallic surface. The profile was fitted to the function $y$ $=a_{1} \sin \left(2 \pi / \lambda_{g}\right)$, where $\lambda_{g}=14.99 \mathrm{~mm}$ and $a_{1}=2.01 \mathrm{~mm}$ provided the best fit. These values compare favorably with those determined via reflectivity measurements. During the process of fitting the reflectivity measurements, both the grating pitch and incident polar angle were used as fitting parameters. Since degeneracy exists between $\theta$ and $\lambda_{g}$, the small deviation between the values of the pitch is not surprising.

Virtually all of the previous experimental studies of SPPs propagating along metal-dielectric interfaces have been undertaken at visible or infrared wavelengths. ${ }^{4,5,7,12,13,20,29}$ At these frequencies the electromagnetic response of the metallic sample is dependent on both the real and imaginary parts of the dielectric function of the substrate, in addition to the interface profile. However, at microwave wavelengths, all metals behave as near-perfect conduc- 
tors and hence the penetration of the electric field into the substrate is small compared to the radiation wavelength. In effect, the electromagnetic response of perfectly conducting gratings is largely independent of their permittivities. The reflectivity is only dependent on the surface profile of the sample, defined in the modeling theory as the sum of a truncated Fourier series, $A(x)$ [Eq. (2)].

The resonant coupling to the SPP is clearly illustrated in Fig. 6. Consider, for example, graph (a). The two peaks correspond to two different coupling mechanisms to the surface mode. The peak at just over $10.5 \mathrm{~mm}$ corresponds to a first order coupling to the +1 SPP (via one grating wave vector, $k_{g}$ ), while the peak at around $8 \mathrm{~mm}$ represents a second order coupling to the -2 SPP via two successive $-k_{g}$ scatterings. Coupling to the -2 SPP is also possible via a single $-2 k_{g}$ interaction. However since the profile of the interface has been milled, and shown above, to be very closely sinusoidal (i.e., the magnitude of the surface profile Fourier coefficients $a_{2}$ and higher, are small compared to the fundamental amplitude, $\left.a_{1}\right)$ the above-mentioned second order interaction dominates. As the azimuthal angle is increased the resonances move towards each other and coalesce at $\varphi=60^{\circ}$, having separated again at $\varphi=75^{\circ}$. Note also the corresponding pseudocritical edges, normally visible in momentum scans undertaken on real metals at visible frequencies ${ }^{12}$ $\left(-\infty \ll \epsilon_{r}<0\right)$ are not visible in these results. This is attributed to two differences between these microwave experiments and those conducted in the visible regime. First, as the frequency is reduced, the SPP dispersion approaches the light line (Fig. 1) hence we find that the momentum difference between the critical edge and the resonance condition is much less than for the visible, making the critical edge difficult to experimentally separate from the surface plasmon resonance. We also suffer from a reduced resolution in momentum due to the angle of incidence spread that is associated with the nature of the source and distances involved in the experiment.

\section{CONCLUSIONS}

In this article, we have provided a thorough experimental investigation of the coupling of microwave energy to, and reradiation of energy from a surface mode propagating on a corrugated metal-dielectric interface. This surface wave has been identified as the surface plasmon polariton, the dispersion of which is determined by the surface profile and the dielectric characteristics of the media either side of the interface. Many previous workers have investigated the experimental excitation of the mode on lossy-metallic gratings at visible and infrared wavelengths. However there is little experimental evidence in the literature regarding the resonant coupling to the SPP that propagates on near-perfectly conducting metals using radiation of the microwave regime. At these frequencies, it is only possible to experimentally observe the resonance of the mode if the corrugation is made sufficiently deep such that the mode's propagation length is reduced.

Experiments at microwave frequencies often suffer from a large amount of beam spread. ${ }^{30}$ However we have devised an experimental arrangement that reduces this problem by collimating the incident beam. We have recorded the specularly reflected signal from a $15-\mathrm{mm}$-pitch metallic grating between 7.5 and $11.3 \mathrm{~mm}$, and have obtained theoretical fits to the reflectivity data of an unprecedented quality.

\section{ACKNOWLEDGMENTS}

The authors would like to acknowledge the financial support of the Defense Evaluation and Research Agency (Farnborough). We would also like to thank Dave Baker and Dr. Lee Bridger (The Polymer Center of the South West, School of Engineering, University of Exeter) for their help in the manufacture of the grating, Steve Tuckett and Pete Cann who built and manufactured various pieces of the apparatus including the mirrors, and Nick Wanstall for his assistance with the grating modeling theory.

${ }^{1}$ H. M. Barlow and A. L. Cullen, Proc. IEE 100, 329 (1953).

${ }^{2}$ G. Goubau, IRE Trans. Antennas Propag. 7, S140 (1959).

${ }^{3}$ J. Zenneck, Ann. Phys. 23, 846 (1907).

${ }^{4}$ R. W. Wood, Philos. Mag. 4, 396 (1902).

${ }^{5}$ H. Raether, Surface Plasmons on Smooth and Rough Surfaces and on Gratings (Springer, Berlin, 1988).

${ }^{6}$ U. Fano, Phys. Rev. 50, 573 (1936).

${ }^{7}$ L. R. Ingersoll, Astrophys. J. 51, 129 (1920).

${ }^{8}$ E. Kretschmann and H. Raether, Z. Naturforsch. A 23, 2135 (1968).

${ }^{9}$ A. Otto, Z. Phys. 216, 398 (1968).

${ }^{10}$ S. J. Elston, G. P. Bryan-Brown, and J. R. Sambles, Phys. Rev. B 44, 6393 (1991).

${ }^{11}$ J. Chandezon, M. T. Dupuis, G. Cornet, and D. Maystre, J. Opt. Soc. Am. 72, 839 (1982).

${ }^{12}$ R. A. Watts, J. R. Sambles, and J. B. Harris, Opt. Commun. 135, 189 (1997).

${ }^{13}$ D. J. Nash and J. R. Sambles, J. Mod. Opt. 43, 81 (1996).

${ }^{14}$ G. N. Zhizhin, M. A. Moskalova, E. V. Shomina, and V. A. Yakovlev, Surface Polaritons: Electromagnetic Waves at Surfaces and Interfaces (North-Holland, Amsterdam, 1982).

${ }^{15}$ M. C. Hutley, Diffraction Gratings (Academic, London, 1982).

${ }^{16}$ W. C. Meecham and C. W. Peters, J. Appl. Phys. 28, 216 (1957).

${ }^{17}$ Lord Rayleigh, Philos. Mag. 14, 213 (1907).

${ }^{18}$ W. C. Meecham, J. Appl. Phys. 27, 361 (1956).

${ }^{19}$ C. H. Palmer, F. C. Evering, and F. M. Nelson, Appl. Opt. 4, 1271 (1965).

${ }^{20}$ I. Pockrand, J. Phys. D 9, 2423 (1976).

${ }^{21}$ R. C. McPhedran and M. D. Waterworth, Opt. Acta 19, 877 (1972).

${ }^{22}$ R. C. McPhedran and M. D. Waterworth, Opt. Acta 20, 533 (1973).

${ }^{23}$ I. Pockrand, Phys. Lett. 49A, 259 (1974).

${ }^{24}$ M. B. Pande and S. D. Gupta, Ind. J. Appl. Phys. 28, 162 (1990).

${ }^{25}$ S. C. Kitson, W. L. Barnes, and J. R. Sambles, Phys. Rev. B 52, 11441 (1995).

${ }^{26}$ J. F. James and R. S. Sternberg, The Design of Optical Spectrometers (Chapman and Hall, London, 1969).

${ }^{27}$ R. A. Watts and J. R. Sambles, Opt. Commun. 140, 179 (1997).

${ }^{28}$ N. P. K. Cotter, T. W. Priest, and J. R. Sambles, J. Opt. Soc. Am. A 12, 1097 (1995).

${ }^{29}$ D. Beaglehole, Phys. Rev. Lett. 22, 708 (1969).

${ }^{30}$ S. A. Khan, N. Q. Dong, and R. E. Burge, Opt. Eng. (Bellingham) 32, 3249 (1993) 